\title{
Effect of obstructive sleep apnea on mitral valve tenting.
}

\author{
Gregg S. Pressman \\ Albert Einstein Medical Center \\ Vincent M. Figueredo \\ Thomas Jefferson University \\ Abel Romero-Corral \\ Albert Einstein Medical Center \\ Ganesan Murali \\ Albert Einstein Medical Center \\ Morris N Kotler \\ Albert Einstein Medical Center \\ Follow this and additional works at: https://jdc.jefferson.edu/cardiologyfp \\ Part of the Cardiology Commons \\ Let us know how access to this document benefits you
}

\section{Recommended Citation}

Pressman, Gregg S.; Figueredo, Vincent M.; Romero-Corral, Abel; Murali, Ganesan; and Kotler, Morris N, "Effect of obstructive sleep apnea on mitral valve tenting." (2012). Division of Cardiology Faculty Papers. Paper 20.

https://jdc.jefferson.edu/cardiologyfp/20

This Article is brought to you for free and open access by the Jefferson Digital Commons. The Jefferson Digital Commons is a service of Thomas Jefferson University's Center for Teaching and Learning (CTL). The Commons is a showcase for Jefferson books and journals, peer-reviewed scholarly publications, unique historical collections from the University archives, and teaching tools. The Jefferson Digital Commons allows researchers and interested readers anywhere in the world to learn about and keep up to date with Jefferson scholarship. This article has been accepted for inclusion in Division of Cardiology Faculty Papers by an authorized administrator of the Jefferson Digital Commons. For more information, please contact: JeffersonDigitalCommons@jefferson.edu. 


\title{
As submitted to: \\ American Journal of Cardiology \\ And later published as:
}

\section{Effect of Obstructive Sleep Apnea on Mitral Valve Tenting \\ Volume 109, Issue 7, 1 April 2012, Pages 1055-1059 \\ 10.1016/j.amjcard.2011.11.037}

\author{
Gregg S. Pressman $\mathrm{MD}^{\mathrm{a}}$, \\ Vincent M. Figueredo $\mathrm{MD}^{\mathrm{a}}$ \\ Abel Romero-Corral MD, MSc ${ }^{\mathrm{a}}$ \\ Ganesan Murali MD ${ }^{b}$ \\ Morris N. Kotler $\mathrm{MD}^{\mathrm{a}}$
}

Department of Medicine, Division of Cardiology ${ }^{a}$ and Division of Pulmonary and Critical Care Medicine ${ }^{\mathrm{b}}$

Albert Einstein Medical Center Philadelphia, PA 19141

Funded by a grant from the Albert Einstein Society, Philadelphia, PA

Correspondence to:

Gregg S. Pressman MD

Albert Einstein Medical Center

5501 Old York Rd, 
Levy Bldg, $3^{\text {rd }}$ Floor

Philadelphia, PA, 19141

Ph: 215-456-8991

Fax: 215-456-3533

Email: PressmanG@einstein.edu 


\section{Abstract}

Obstructive apneas produce high negative intrathoracic pressure which imposes an afterload burden on the left ventricle. Such episodes might produce structural changes in the left ventricle over time. Doppler echocardiograms were obtained within 2 months of attended polysomnography. Patients were grouped according to apnea-hypopnea index (AHI): mild/no OSA (AHI < 15) and mod/severe OSA (AHI $\geq$ 15). Mitral valve tenting height and area, left ventricular (LV) long and short axis, and LV end-diastolic volume (LVEDV), were measured along with tissue Doppler parameters. Comparisons of measurements at baseline and follow up between and within groups were obtained; correlations between absolute changes (deltas) in echocardiographic parameters were also performed. After a mean follow up of 240 days mitral valve tenting height increased significantly $(1.17 \pm 0.12 \mathrm{~cm}$ to $1.28 \pm 0.17 \mathrm{~cm}, \mathrm{p}=0.001)$ in $\mathrm{mod} / \mathrm{severe}$ OSA as did tenting area $\left(2.30 \pm 0.41 \mathrm{~cm}^{2}\right.$ to $\left.2.66 \pm 0.60 \mathrm{~cm}^{2}, \mathrm{p}=0.0002\right)$; delta tenting height correlated with delta LVEDV (rho $0.43, \mathrm{p}=0.01$ ) and delta tenting area (rho $0.35, \mathrm{p}=0.04$ ). In mild/no OSA patients there was no significant change in tenting height; there was a borderline significant increase in tenting area $\left(2.20 \pm 0.44 \mathrm{~cm}^{2}\right.$ to $\left.2.31 \pm 0.43 \mathrm{~cm}^{2}, \mathrm{p}=0.05\right)$. Septal $\mathrm{E}^{\prime}$ decreased $(8.04 \pm 2.49 \mathrm{~cm} / \mathrm{sec}$ to $7.10 \pm 1.83 \mathrm{~cm} / \mathrm{sec}, \mathrm{p}=0.005$ ) in $\mathrm{mod} / \mathrm{severe}$ OSA subjects, but not in the mild/no OSA group. In conclusion, in patients with mod/severe OSA, mitral valve tenting height and tenting area increase significantly over time. This appears to be related, at least in part, to changes in LV geometry.

Running Head: OSA increases mitral valve tenting

Key Words: Mitral valve tenting, obstructive sleep apnea, left ventricular geometry 


\section{Introduction}

Obstructive sleep apnea (OSA) has adverse effects on left ventricular (LV) diastolic function ${ }^{1}$ and on global LV function ${ }^{2-4}$. However, aside from producing increased wall thickness ${ }^{2}$ there is little information available regarding effects on left heart structure. We tested the hypothesis that moderate to severe OSA, over time, can lead to changes in mitral valve tenting height and area in association with changes in LV geometry.

\section{Methods}

This prospective longitudinal study recruited subjects from the Sleep Disorders Center at Albert Einstein Medical Center between January 2008 and May 2010. All had sleep tests ordered on clinical grounds and none had a previously diagnosed sleep disorder. Exclusions included 1) any evidence of coronary disease, 2) mitral leaflet disease, and 3) decreased ejection fraction for any reason.

Demographic and clinical data were collected but no attempt was made to control medical therapy. Subjects underwent Doppler echocardiography shortly after polysomnography and again 6 months or more after the initial study. For those prescribed continuous positive airway pressure, compliance was measured by interrogation of the device or "smartcard". Because only 4 subjects were compliant (> 4 hours/night), the current paper reflects the natural history of untreated severe OSA.

Polysomnography was performed utilizing the VIASYS SomnoStar Pro Sleep System (SomnoStar Software 9-1b). The electroencephalogram, electro-oculogram, and electromyogram were monitored for sleep staging. Nasal pressure monitoring, chest wall movement, abdominal movement, snoring and oxygen saturation were recorded for respiratory assessment. The electrocardiogram and tibial 
electromyogram were monitored for cardiac arrhythmias and nocturnal limb movements respectively. Studies were interpreted according to the standards of the American Academy of Sleep Medicine . $^{5}$ Presence and severity of OSA were established based on apnea-hypopnea index (AHI) : $<5=$ no OSA, $\geq 5$ $\mathrm{AHI}<15=$ mild OSA,$\geq 15 \mathrm{AHI}<30=$ moderate OSA, and $\mathrm{AHI} \geq 30=$ severe OSA. Interpretation of all polysomnograms was performed by board certified sleep medicine physicians.

Two-dimensional and Doppler echocardiography were performed according to American Society of Echocardiography standards ${ }^{6}$. Mitral valve tenting area was measured in mid-systole in the parasternal long-axis view as the area bounded by the closed mitral leaflets and a line connecting the annular attachment points of the 2 leaflets. Tenting height was defined as the distance from that line to the point of coaptation of the leaflets ${ }^{7}$ (figure 1). Left atrial dimension was measured as the diameter of the left atrium in systole in the parasternal long-axis view. Left atrial volume was measured in the apical views according to the biplane area-length method. The LV long axis was measured at end-diastole from the annular plane to the apex in the 4-chamber view. The LV short axis was measured in the same view as the diameter of the left ventricle (endocardium to endocardium) at the tips of the open mitral valve leaflets. LV end diastolic volume (LVEDV) and end systolic volume (LVESV) were measured using the modified Simpson's biplane method. The interpapillary muscle distance of the left ventricle was measured at end-diastole in the parasternal short axis view by drawing a line through the papillary muscles and measuring between the LV endocardial borders where the papillary muscles attached. Tissue Doppler measurements included the systolic wave $\left(S^{\prime}\right)$ and early diastolic wave ( $\left.E^{\prime}\right)$ measured at the septal and lateral aspects of the mitral valve annulus in the 4-chamber view.

Two experienced, board certified echocardiographers (GSP and VMF) read all the echocardiograms. In order to avoid interobserver variability, each parameter was measured by only one 
observer (GSP: tenting height, tenting area, LVEDV, LVESV, left atrial dimension, left atrial volume; VMF: LV long axis, LV short axis, LV interpapillary muscle distance, $E^{\prime}$, and $\left.S^{\prime}\right)$. Values for each measured parameter were obtained by averaging a minimum of 3 measurements for that parameter. Echocardiograms were read in a blinded manner without knowledge of OSA status.

Continuous variables are displayed as mean \pm standard deviation and categorical variables are displayed as numbers and percentages. Paired analyses were used to compare variables within groups over time. Non-parametric correlations (Spearman) were used to assess the relationship between absolute changes (deltas) in echocardiographic variables over time in both groups. Two-tailed $p$ values $<$ 0.05 were considered significant in advance. Linear regression was used to assess for univariate predictors of change in tenting height and tenting area; those variables with $p$ values $<0.10$ were included in multivariate analysis. Multivariate linear regression was used to assess for predictors of change in tenting height and area after adjustment for variables identified in the univariate analyses; $p$ values $<0.05$ were considered significant. Intraobserver variability was calculated for tenting height and tenting area, expressed as percentages. All analyses were performed using JMP 8.0 software.

\section{Results}

Fifty-four subjects were enrolled and followed for a mean of 240 days (range 180 - 431). Six patients did not have follow-up echocardiograms ( $11 \%$ of the sample). Baseline characteristics of the subjects included in the study are displayed in table 1. 
When divided into 2 groups, $\mathrm{AHI}<15$ (mild/no OSA) and $\mathrm{AHI} \geq 15$ (mod/severe OSA), baseline characteristics were similar between groups except for BMI which was higher in the group with moderate to severe OSA.

Intraobserver variability for the main outcome variables was $4 \%$ for tenting height and $6 \%$ for tenting area. Baseline Doppler echocardiographic variables for the $2 \mathrm{AHI}$ groups are presented in table 2. Only lateral E' differed significantly between groups $(p=0.05)$. Table 3 presents Doppler echocardiographic variables over time stratified by AHI groups. Of note, among those with mod/severe OSA, tenting height increased over time $(p=0.001$; figure $2 a)$ as did tenting area $(p=0.0002$; figure $2 b)$. In the group with mild/no OSA there was no significant change in tenting height while a borderline significant increase in tenting area $(p=0.05)$ was observed. Septal $E^{\prime}$ decreased significantly among $\mathrm{mod} / \mathrm{severe}$ OSA, with no significant change noted in those with mild/no OSA.

We also performed correlations within the $2 \mathrm{AHI}$ groups to test whether changes in tenting height and tenting area were dependent on changes in LV geometry. No significant correlations were found in the mild/no OSA group. However, among mod/severe OSA subjects, delta tenting height had a positive and significant correlation with delta LV long axis (rho $0.39, p=0.02$ ), delta LV short axis (rho 0.36, $p=0.03$ ), and delta LVEDV ( rho $0.43, p=0.01$; figure 3 ). A positive and significant correlation between delta tenting area and delta LVEDV (rho 0.35, $p=0.04$ ) was also observed; however, delta LV long axis and delta LV short axis showed nonsignificant, though positive, correlations.

Linear regression analysis assessing for univariate predictors of change in tenting height showed only $\mathrm{AHI}$ to be a significant predictor ( $\mathrm{F}$ ratio $3.83, \mathrm{p}=0.05$ ). Furthermore, $\mathrm{AHI}$ remained a significant predictor of tenting height on multivariate analysis ( $F$ ratio 3.93, $p=0.04$ ) after adjustment for age, 
gender, race, BMI and hypertension. No significant predictors were found for tenting area $(p>0.10$ for each variable).

It should be noted that, despite changes in tenting height an area over time, no subject developed significant mitral regurgitation.

\section{Discussion}

The main finding of this study is that tenting height and tenting area increased significantly over time in patients with moderate to severe OSA. This appeared to be related, in part, to remodeling of the left ventricle. Obstructive apneas impose an afterload burden on the heart ${ }^{8-10}$ and can occur hundreds of times per night. The cumulative effects of this pathophysiology, over months to years, might account for changes in mitral valve tenting and LV chamber dimensions.

It is generally accepted that changes in ventricular shape can produce changes in tenting height and tenting area. In disease states that produce LV dilation, tenting is associated with outward and apical displacement of the papillary muscles and often produces clinically significant mitral regurgitation 7,11,12. In this setting, the ventricle assumes a more spherical shape. Changes in LV geometry are also commonly seen in athletes although associated mitral regurgitation has not been reported. As opposed to disease states, ventricular shape appears to be maintained in athletes though chamber dimensions increase ${ }^{13-16}$. The current data suggest similar changes in LV geometry in patients with moderate to severe OSA. 
The finding that OSA increases mitral valve tenting is potentially important in several ways. While none of our subjects developed clinically significant mitral regurgitation, it is conceivable that such might develop over a longer time period. The current study excluded subjects with dilated ventricles and associated mitral regurgitation. It may be that OSA worsens pre-existing mitral regurgitation by increasing tenting. It is also possible that significant mitral regurgitation only occurs during obstructive episodes when blood pressure and afterload are high. Our group has shown that following an apneic episode (simulated by the Mueller maneuver), there is a compensatory increase in blood flow to the left side of the heart, which could augment transient mitral regurgitation. ${ }^{9}$ In fact, most episodes of acute pulmonary edema, where mitral regurgitation may be an important contributing factor, occur at night.

Few previous studies have looked at effects of OSA on LV geometry. Nearly all are observational and cross-sectional in nature. Dursunoglu et al measured end diastolic dimension by M-mode echocardiography in 67 subjects with OSA who had no cardiac disease ${ }^{17}$. They found a non-significant trend towards higher values as $\mathrm{AHI}$ increased. Drager et al studied 60 patients with hypertension and/or OSA in whom other disorders were carefully excluded ${ }^{18}$. Comparing normotensive subjects with OSA to controls, they found no significant difference in LV end diastolic dimension between groups. However, in another study of OSA and hypertension, Myslinski et al observed significantly higher values of LV end diastolic dimension in OSA subjects with adequately treated hypertension when compared with controls 19.

In addition to changes in mitral valve tenting, this study documented a decrease over time in the tissue Doppler parameter E' among those with moderate to severe OSA. This is consistent with previous 
reports ${ }^{1,2,20}$. It is important as decreased $E^{\prime}$ is a marker of worsening of global LV diastolic function, which in turn is associated with heart failure, pulmonary hypertension, and atrial fibrillation.

This study involved a small number of patients and the findings need to be reproduced in a larger sample. None of the subjects developed significant mitral regurgitation and any connection between LV remodeling, tenting height and area, and development of future mitral regurgitation remains speculative. However, increases in tenting height and area have been strongly associated with mitral regurgitation in diseased ventricles. In addition, these studies were carried out in conscious subjects. It is possible that mitral regurgitation occurs during an obstructive apnea but not in the waking state. One of the limitations of OSA research generally, is the difficulty in imaging the heart during an obstructive apnea.

Our study sample was largely minority and thus our findings might not apply to the general population. However, there is no reason to believe that the findings would vary by race or ethnicity. More likely, they are the result of the physiologic perturbations associated with obstructive sleep apnea.

The major strengths of this study are its prospective nature and the blinded interpretation of the echocardiograms. In addition, the increases in mitral valve tenting height and tenting area were associated with changes in LV geometry which could plausibly explain them. 


\section{Figure Legends}

Figure 1. Parasternal long axis view in midsystole. The mitral valve tenting area is measured by drawing a line connecting the annular attachment points of the mitral leaflets and then tracing the leaflet borders. In this case the tenting area is $2.1 \mathrm{~cm}^{2}$ (measurement algorithm also indicates the length of the border, $8.0 \mathrm{~cm}$ ). The tenting height is measured by drawing a perpendicular line from the annular line to the point of leaflet coaptation, in this case $1.3 \mathrm{~cm}$.

Figure 2. Mitral valve tenting height (2a) and tenting area ( $2 b)$ at baseline and at follow-up in the $\mathrm{mild} / \mathrm{no}$ OSA and mod/severe OSA groups. Both variables are seen to increase significantly over time in the mod/severe OSA group.

Figure 3. Scatterplot of change in mitral valve tenting height as a function of change in left ventricular end diastolic volume (biplane modified Simpson's method). Rho $=0.42$ indicating a good correlation and suggesting that change in left ventricular end diastolic volume accounts for at least some of the change in mitral valve tenting height. 
1. Arias MA, Garcia-Rio F, Alonso-Fernandez A, Mediano O, Martinez I, Villamor J. Obstructive sleep apnea syndrome affects left ventricular diastolic function: effects of nasal continuous positive airway pressure in men. Circulation 2005;112:375-383.

2. Shivalkar B, Van de Heyning C, Kerremans M, et al. Obstructive sleep apnea syndrome: more insights on structural and functional cardiac alterations, and the effects of treatment with continuous positive airway pressure. J Am Coll Cardiol 2006;47:1433-1439.

3. Romero-Corral A, Somers VK, Pellikka PA, et al. Decreased right and left ventricular myocardial performance in obstructive sleep apnea. Chest 2007;132:1863-1870.

4. Koga S, Ikeda S, Urata J, Kohno S. Effect of nasal continuous positive airway pressure in men on global left ventricular myocardial performance in patients with obstructive sleep apnea syndrome. Am J Cardiol 2008;101:1796-1800.

5. The AASM Manual for the Scoring of Sleep and Associated Events: Rules, Terminology and Technical Specification. Westchester, III.: The American Academy of Sleep Medicine; 2008.

6. Lang RM, Bierig M, Devereux RB, et al. Recommendations for chamber quantification: a report from the American Society of Echocardiography's Guidelines and Standards Committee and the Chamber Quantification Writing Group, developed in conjunction with the European Association of Echocardiography, a branch of the European Society of Cardiology. J Am Soc Echocardiogr 2005;18:1440-1463.

7. Yiu SF, Enriquez-Sarano M, Tribouilloy C, Seward JB, Tajik AJ. Determinants of the degree of functional mitral regurgitation in patients with systolic left ventricular dysfunction: A quantitative clinical study. Circulation 2000;102:1400-1406.

8. Robotham JL, Lixfeld W, Holland L, MacGregor D, Bryan AC, Rabson J. Effects of respiration on cardiac performance. J Appl Physiol 1978;44:703-709. 
9. Buda AJ, Pinsky MR, Ingels NB, Jr., Daughters GT, 2nd, Stinson EB, Alderman EL. Effect of intrathoracic pressure on left ventricular performance. N Engl J Med 1979;301:453-459.

10. Orban M, Bruce CJ, Pressman GS, et al. Dynamic changes of left ventricular performance and left atrial volume induced by the mueller maneuver in healthy young adults and implications for obstructive sleep apnea, atrial fibrillation, and heart failure. Am J Cardiol 2008;102:1557-1561.

11. Boltwood CM, Tei C, Wong M, Shah PM. Quantitative echocardiography of the mitral complex in dilated cardiomyopathy: the mechanism of functional mitral regurgitation. Circulation 1983;68:498-508.

12. Kono $\mathrm{T}$, Sabbah $\mathrm{HN}$, Rosman $\mathrm{H}$, et al. Mechanism of functional mitral regurgitation during acute myocardial ischemia. J Am Coll Cardiol 1992;19:1101-1105.

13. Karjalainen J, Mantysaari M, Viitasalo M, Kujala U. Left ventricular mass, geometry, and filling in endurance athletes: association with exercise blood pressure. J Appl Physiol 1997;82:531-537.

14. Pelliccia A, Maron BJ, De Luca R, Di Paolo FM, Spataro A, Culasso F. Remodeling of left ventricular hypertrophy in elite athletes after long-term deconditioning. Circulation 2002;105:944-949.

15. Fagard R. Athlete's heart. Heart 2003;89:1455-1461.

16. De Castro S, Caselli S, Maron M, et al. Left ventricular remodelling index (LVRI) in various pathophysiological conditions: a real-time three-dimensional echocardiographic study. Heart 2007;93:205-209.

17. Dursunoglu D, Dursunoglu N, Evrengul H, et al. Impact of obstructive sleep apnoea on left ventricular mass and global function. Eur Respir J 2005;26:283-288.

18. Drager LF, Bortolotto LA, Figueiredo AC, Silva BC, Krieger EM, Lorenzi-Filho G. Obstructive sleep apnea, hypertension, and their interaction on arterial stiffness and heart remodeling. Chest 2007;131:1379-1386. 
19. Myslinski W, Duchna HW, Rasche K, Dichmann M, Mosiewicz J, Schultze-Werninghaus G. Left ventricular geometry in patients with obstructive sleep apnea coexisting with treated systemic hypertension. Respiration 2007;74:176-183.

20. Otto ME, Belohlavek M, Romero-Corral A, et al. Comparison of cardiac structural and functional changes in obese otherwise healthy adults with versus without obstructive sleep apnea. Am J Cardiol 2007;99:1298-1302. 